\title{
Diagnostic reasoning in osteopathy $e$ A qualitative study
}

\author{
Oliver P. Thomson, Nicola J. Petty, Ann P. Moore
}

KEYWORDS

Clinical reasoning; Osteopathy; Diagnosis; Manual therapy; Decision making

\begin{abstract}
Background: The clinical reasoning processes which result in the forma- tion of a diagnosis, are fundamental for safe, effective and efficient clinical prac- tice and are central to professional autonomy and accountability. While research has identified the diagnostic reasoning approaches taken by a range of healthcare professions, there is limited understanding of how osteopaths formulate diagnoses in clinical practice.

Objectives: The aim of this research was to explore the diagnostic reasoning of experienced osteopaths in the UK.

Methods: A qualitative constructivist grounded theory approach was taken in this study, which was situated in the interpretive research paradigm. A total of seven- teen face-to-face semi-structured interviews were conducted with twelve experi- enced osteopaths. Participants were purposefully and theoretically sampled to take part in this study. Data collection methods involved semi-structured interviews with participants and observation and video-recording of clinical appointments, which were followed by video-prompted reflective interviews. The constant comparative method of analysis was used to code and analyse data.

Results: The findings suggest that when formulating a diagnosis practitioners adopted two diagnostic reasoning approaches, namely hypothetico-deductive reasoning and pattern recognition. In this study, there was interplay of these reasoning approaches as a result of the perceived level of complexity and degree of familiarity of the patient presentation.

Conclusions: Experienced osteopaths adopted diagnostic reasoning approaches which are akin to other healthcare professions including medicine and physio- therapy. Metacognitive and refl xivity skills were central for safe and effective diagnostic reasoning. Further work is required to explore the transferability of these findings with practitioners of different levels of clinical experience and who work in different clinical settings.
\end{abstract}




\section{Introduction}

Clinical reasoning refers to the social, cognitive and interactive processes by which practitioners make decisions in practice. ${ }^{1}$ Specifically, diagnostic reasoning refers to the reasoning processes which result in the formation of a diagnosis, often related to patients' physical disability and impairment with consideration of associated pain mechanisms, tissue pathology, and potential and wide-ranging contributing factors. ${ }^{2}$ Researchers across the healthcare professions have been attempting to understand the processes of clinical reasoning for more than forty-five years, with the majority of the research focusing primarily on the process practitioners use to arrive at a diagnosis. ${ }^{3}$

\section{Approaches to clinical reasoning research}

Since clinical reasoning is a social and cognitive process and therefore resides in the 'head' of the practitioner, largely hidden from observers ${ }^{4}$ this poses a challenge for researchers wanting to explore this area of clinical practice. A range of research methods, operating in different research paradigms, have been used to investigate the different aspects of clinical reasoning. ${ }^{4,5}$
Throughout the last five decades, there have been notable trends in the methodologies and theories of clinical reasoning research. The medical profession were amongst the first to actively research clinical reasoning and have strongly influenced the reasoning approaches adopted in other health professions. ${ }^{5}$ Research into clinical reasoning has used both qualitative and quantitative methodological approaches to data gathering and analysis. Early medical researchers of clinical reasoning in the 1970 s operated predominantly in the positivist/ post-positivist paradigm, using largely quantitative research approaches. ${ }^{6}$ However, more recent clinical reasoning research in other healthcare professions including physiotherapy, ${ }^{2,7,8}$ occupational therapy ${ }^{9 e 11}$ and nursing ${ }^{12 \mathrm{e} 14}$ has operated within the interpretive research paradigm, using qualitative methodologies.

Quantitative approaches are well suited to limit, test or measure specific aspects of clinical reasoning (for example, 'do experts or novices generate more differential diagnoses?'), while qualitative methods will illuminate factors which can help to explain and understand an individual's clinical reasoning (for example, 'how do practitioners structure their treatment and management plan?'). ${ }^{3}$ Whether taking a quantitative or qualitative approach, observation and/or videorecording of treatment sessions, interviews with practitioners and patients, and written materials from the practitioners (for example, reflective diaries) have served as data collection methods in clinical reasoning research, all with varying strengths and weaknesses (for an overview see Unsworth4). 


\section{The development of diagnostic reasoning research}

In the late 1970s, researchers attempted to gain an understanding of the diagnostic reasoning processes used within medicine. Using students of different educational levels ${ }^{15}$ or a combination of students and physicians, ${ }^{16}$ these studies observed participants' interaction with patients during a clinical appointment. Participants then gave a real-time verbal description, either during the

interaction ('think aloud') or alternatively after the patient encounter, whilst also viewing a videorecording of the clinical appointment, termed 'stimulated recall'. Drawing on previous research comparing the performance of master and novice chess players, ${ }^{17,18}$ Elstein and colleagues ${ }^{19}$ took an observational approach to study diagnostic reasoning, by investigating how 'expert' ${ }^{\mathrm{d}}$ medical practitioners solved clinical problems and arrived at a diagnosis. The results of these studies showed that expert practitioners began to formulate multiple hypotheses early on in the patient encounter and that the diagnostic accuracy was closely related to previous exposure and experience of specific types of patient cases. The key finding of Elstein's study was that diagnostic reasoning involved four steps: 1) data or 'cue' acquisition (initial observation, case history, examination findings); 2) hypothesis formation; 3) interpretation of the data ('weighing of evidence'); followed by 4) a search to prove (or disprove) the evidence (hypothesis evaluation). ${ }^{19}$ What emerged from Elstein's seminal work was an early general model of medical diagnostic reasoning, termed the 'hypothetico-deductive' method. ${ }^{24}$

Later, in the 1980s, medical researchers began focusing on the different ways in which expert physicians organised and structured their knowledge in relation to their diagnostic reasoning. Groen and Patel $^{25}$ identified that during non-problematic situations, experts did not rely solely on hypothetico-

\footnotetext{
d The concept of the 'expert' is problematic, and researchers currently have a limited understanding of the nature of expertise in healthcare (for example see, Mylopoulos et al. ${ }^{20}$ Jensen et al. ${ }^{21,22}$ and Petty et al..$^{23}$ ).
}

deductive reasoning, but rather used direct retrieval of information from a well-developed knowledge base to support their analysis of presenting data and subsequent hypothesis generation, termed 'pattern recognition'. Pattern recognition, also known as 'forward reasoning', contrasts with the cyclical process of hypothesis generation and testing (also referred to as 'backward reasoning'). Patel and Groen ${ }^{25}$ proposed that novices tend to use processes such as means-ends analysis and generate-and-test methods that involve slower backward reasoning. Barrows and Feltovich ${ }^{26}$ posited that practitioners use hypotheticodeductive reasoning as a means to store new clinical patterns in their memory which may be relied upon when a similar clinical situation arises.

Further research has suggested that when confronted with a complex or unfamiliar problem, expert medical practitioners resort back to hypothetico-deductive reasoning. ${ }^{26 e 28}$ Although efficient, pattern recognition can be error prone and reducing the hazards associated with pattern recognition requires a strong domain specific knowledge and metacognitive processes. Jones and colleagues highlight how to avoid the potential hazards of pattern recognition and state that "learning and being able to recognise common clinical patterns and their variations while minimising the risks and limitations of pattern recognition requires metacognition". ${ }^{29, p .250}$ The ability of the practitioner to self-reflect and "know what they know and what they don't know' affords them the ability to monitor their data collection and is thought to be ${ }_{21,29}$ vital part of expert practice in physiotherapy.

Since the seminal clinical reasoning studies of the 1970s and 1980s, research has shown that hypothetico-deductive reasoning and pattern recognition approaches are core strategies in the

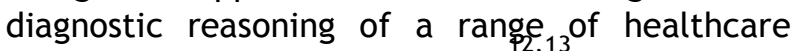
professionals, including nurses, 2,7,8, $\}_{31}, 32$ ational therapists and physiotherapists.

\section{Diagnostic reasoning and osteopathy}

In the UK and the antipodes, osteopaths are autonomous manual therapy professionals who require a broad ranging knowledge and skill base in order to diagnose, treat and manage patients with a variety of musculoskeletal and non-musculoskeletal conditions which present in clinical practice. ${ }^{33,34}$ With regards to presenting symptoms, spinal pain is by far the most common condition treated by osteopaths in the UK. ${ }^{33,34}$ Practitioners employ a broad spectrum of therapeutic interventions, with manipulative 
techniques (such as spinal mobilisation and highvelocity thrust manipulation) as the preferred form of treatment modalities for practitioners in the $\mathrm{UK}^{33 e 35}$ as well as internationally. ${ }^{36,37}$ For example, a 2001 survey of the UK osteopathic profession conducted by the $\mathrm{GOsC},{ }^{33}$ showed that almost $75 \%$ of responders regularly used joint mobilisation techniques, and almost $50 \%$ regularly used high-velocity thrust techniques, as part of their treatment, and similar statistics are supported by more recent research. ${ }^{34,35}$

In view of the wide range of treatment interventions employed by practitioners and the diversity and complexity of problems that patients present with in clinical practice, well developed clinical reasoning skills are essential to osteopaths. Specifically, diagnostic reasoning is fundamental to safe and effective osteopathic practice and is central to professional autonomy and accountability. Furthermore, the current standards of osteopathic practice demand that practitioners in the UK are able to evaluate patients effectively in order to "make a working diagnosis and formulate a treatment plan".38,p.13 The most recent standards of practice set out by the GOsC explicitly state that osteopaths are required to have "problem-solving and thinking skills in order to inform and guide the interpretation of clinical and other data, and to justify clinical reasoning and decisionmaking". 38, p.9 Similar competencies in diagnostic reasoning are echoed by the osteopathic regulators in Australia, ${ }^{39} \mathrm{New}$ Zealand ${ }^{40}$ and are found in benchmarks for osteopathic training worldwide. ${ }^{41}$ Although several osteopathic models of diagnostic reasoning models have been proposed theoretically ${ }^{42 e 44}$ there is little-to-no published research exploring the diagnostic reasoning of osteopaths. Therefore a knowledge gap exists between the diagnostic reasoning skills espoused and stipulated by osteopathic regulators and the actual diagnostic reasoning skills of practitioners which take place in-action, during real-world clinical practice.

Research from a range of healthcare professions has shown that well developed diagnostic reasoning skills are fundamental to clinical expertise (for example in physiotherapy ${ }^{2,21,45,46}$ and medicine $^{47 e 49}$ ). Developing a research-based knowledge of the diagnostic reasoning processes used by osteopaths would be valuable to educators and practitioners and ultimately help enhance patient care. ${ }^{3}$ Finally, issues surrounding the nature, uniqueness and role of osteopathy in modern-day healthcare are continually debated by the profession internationally (see for example the Special Edition of this journal on osteopathic principles ${ }^{50}$ ); therefore research which investigates how osteopaths make clinical and diagnostic decisions is able to generate knowledge which sheds light on these fundamental areas of professional practice.

This paper explores the diagnostic reasoning of experienced osteopaths and the findings from this study form part of a broader theory of clinical decision-making and therapeutic approaches reported elsewhere. ${ }^{51 e 54}$

\section{Methods}

\section{Study design}

A constructivist grounded theory ${ }^{55}$ approach was taken in this qualitative study, which was situated in the interpretive research paradigm. A total of seventeen face-to-face semi-structured interviews were conducted with twelve experienced osteopaths, three of which took place immediately after observation of a clinical appointment which was also video-recorded. These three reflective interviews were prompted by the video-recording, which facilitated participants' to reflect more deeply on their reasoning and helped to ensure the interview discussion was firmly grounded in their actions, reasoning and decisions, which took place during the clinical appointment. ${ }^{56}$

In line with grounded theory, data collection and analysis occurred in parallel, in an iterative fashion. ${ }^{55}$ The constant comparative method of analysis was used to code and analyse interview data. ${ }^{55}$ This method of analysis involved the primary research (OT) comparing data with data, data with category, category with category and facilitated the active construction of codes and categories with increasing level of abstraction. ${ }^{55}$ Memos were written throughout the study data collection and analysis, and helped to identify and define codes, patterns and relationships in the data as well as encouraging researcher reflexivity. ${ }^{55}$ Data collection and analysis continued until no new analytical insights became apparent, suggesting theoretical sufficiency had been attained. ${ }^{55}$

Table 1 provides examples of interview questions which were used to explore participants' diagnostic reasoning. All interviews were audiorecorded and transcribed verbatim. The methods used in this study have been presented in detail elsewhere. ${ }^{51,53,54}$

\section{Participants}

A total of twelve UK registered osteopaths were purposefully and theoretically sampled to take 
Table 1 Example of interview questions used to explore participants' diagnostic reasoning.

Imagine we are in your clinic, and you are about to see a new patient. Let's say it's a patient with back pain. Please take me through your thinking process, as you work out what's wrong with this patient.

As you do this test/examination etc what are you focusing on? What are you thinking about? How does this help you decide what's causing the patients problem?

Has the patients' condition met with your expectations? How does this make you feel/think? What were your main concerns about this patient? Did anything take you by surprise while caring for the patient? How do you feel in that situation? How did you know it was safe to proceed with this patient?

part in this study. Participants were recruited from osteopathic educational institutions and through adverts placed in the national osteopathic press. Participants' biographical information is provided in Table 2. Approval was granted by the Faculty of Health and Social Science Research Ethics and Governance Committee at the University of Brighton and the Ethics Committee of the British College of Osteopathic Medicine.

\section{Findings}

A major goal of all participants was establishing an explanation of the patient's problem. All participants initially sought to differentiate between a serious pathology that would require referral to a medical practitioner and a condition that was within their expertise and scope of practice. All participants appeared to spend time focusing on patient's manifestations of disease and dysfunction, (i.e. clinical signs and symptoms) with the aim of measuring and defining the patient's condition. For clarity, the diagnostic reasoning process is presented in the following three stages; however the actual process was iterative across all three:

- Focusing on signs and symptoms

- Generating and testing hypotheses

- Recognising patterns

\section{Focusing on signs and symptoms}

When reasoning diagnostically, participants' attention was focused on patients' signs and

\begin{tabular}{|c|c|}
\hline Mean age & 43.5 (range $30 e 56)$ \\
\hline $\begin{array}{l}\text { Mean years in } \\
\text { clinical practice }\end{array}$ & 15 (range $6 \mathrm{e} 25$ ) \\
\hline Gender & $\begin{array}{l}10 \text { males } \\
2 \text { females }\end{array}$ \\
\hline Work setting & $\begin{array}{l}\text { All worked in private clinical } \\
\text { practice } \\
10 \text { held additional roles as } \\
\text { clinical tutors or lecturers at } \\
\text { an OEI }\end{array}$ \\
\hline Education & $\begin{array}{l}\text { All had undergraduate } \\
\text { qualifications in osteopathy } \\
\text { (BSC, DO) } \\
4 \text { held additional } \\
\text { postgraduate degrees (MSc) }\end{array}$ \\
\hline
\end{tabular}

OEI e Osteopathic Educational Institution; BSc e Bachelor of Science; DO e Diploma in Osteopathy; MSc e Master of Science.

symptoms, and through specific clinical action, such as examination procedures, they sought to acquire cues which would help to explain the cause of the patient's problem. All participants were interested in the precise nature and behaviour of patients' symptoms such as the location of pain, type of pain, and whether there were any associated symptoms. These initial cues formed the basis of ideas or hypotheses about what might be the cause of the patients' problem. These hypotheses were tested by specific questioning, or performing precise procedures and actions (such as clinical testing and examination). These actions produced additional cues which allowed possible hypotheses to be refined, accepted or discarded:

I look for associated symptoms, and I try, with the best possible attempt, to pinpoint dysfunction or tissue causing symptoms, or perhaps more centrally maintained pain states. (P3)

If they've [the patient] come in with left-sided chest or heart problems, l'd be doing a cardiovascular screening, so I always test the most dangerous things first. (P5)

At the centre of participants' thinking were the presenting signs and symptoms, results of clinical tests, questions, and thoughts regarding possible hypotheses regarding a causal explanation of the patients' problem. When focusing on the signs and symptoms of the patient's presenting problem, participant's thinking was directed predominately towards determining causeeeffect relationships, the meaning of biomedical cues, and developing 
hypotheses. It appeared that the constructed diagnosis was the result of participants' own interpretation of the patient's signs and symptoms:

The elements of the case history, the nature of the onset of the pain, diurnal variation, and those kinds of things will tend to lead me towards implicating certain tissues perhaps more than others. (P7)

If the person's presenting with paresthesia in the limb or in the hands or the feet I want to know which nerve root is involved. (P8)

Having focused on the presenting signs and symptoms to acquire cues in order to help explain the patient's condition, practitioners sought to obtain an explanation of the patient's condition which they considered to be 'correct' and 'true'. During the process of diagnosis construction, participants appeared to adopt one of two different diagnostic reasoning approaches; hypotheticodeductive reasoning ${ }^{19}$ or pattern recognition, ${ }^{57}$ and are presented below.

\section{Generating and testing hypotheses}

For all participants, focusing on patients' signs and symptoms during examination procedures enabled them to acquire cues which would help develop hypotheses and guide further examination and treatment. Initial cues formed the basis of ideas or hypotheses about what might be the cause of patients' condition, and all participants directed their reasoning towards 'sieving out' a potentially serious cause of patients' symptoms:

I'm trying to decide "bi-lateral leg pain- where does that leave me"? And I am thinking "is there some sort of aneurysm? Is it degeneration on both sides of his spine? Is there a normal reason for that going on?" (P12)

I make sure that I do my best to rule out anything that I shouldn't [treat] e I'm working with my fear that I don't want to start treating pathology or something non-musculoskeletal. (P6)

Several participants commented that their hypotheses were evaluated by deliberately acquiring further cues via specific questioning and clinical examination procedures, which resulted in hypotheses being modified, accepted or rejected:

In my head I have a rolling selection of differential diagnoses that I aim to rule out with clinical investigation. (P3)
If the patient was complaining of posterior thigh pain, I want to determine what I think is the structure and where that is being compromised in its function. (P8)

When faced with unfamiliar symptoms, several participants commented that they employed a methodical and purposeful process of collecting clinical information, developing and evaluating their hypotheses. This 'step-by-step' process tended to occur when they were confronted with a challenging, complex or unfamiliar problem, and two participants commented:

I rarely now write out a list of differentials, sometimes I do when I'm really stumped. If I really haven't got a good clue, then I spend a few seconds just thinking what it could be, and write it out. (P2)

I'm thinking about maybe it's something systemic, have I missed anything in the prostate, in the abdomen and anything else gynaecologically?. [So] I need to really think about why the back pain isn't getting any better. (P5)

\section{Recognising patterns}

All participants formulated ideas and hypotheses about the cause of the patient's problem by obtaining symptom-based cues during their examination and history taking. At other times, participants appeared to recognise clusters of cues and the relationships between symptoms, suggesting they related these patterns of cues to previous clinical experiences, allowing them to 'jump' forward to a diagnosis. For example, some participants recognised clusters of biomechanicallyrelated cues during physical examination of the patient's body and swiftly arrive at a diagnosis:

Immediately I could see why the neck wasn't allowing that movement and why he was hesitant to do that movement. And it [the diagnosis] was quite frankly already there when he sat there. (P11)

Several participants commented on the ease of which they could arrive at a diagnosis, especially when they encountered a patient with a problem which appeared simple or similar to previous experiences:

I am eighty per cent of the way there just after the case history and questioning, and usually that twenty per cent confirmation takes place during the rest of the time.You build up a better 
repertoire of remembering pain patterns and onset causes and histories, and so on. And that back catalogue of data helps you get to an answer quickly. (P4)

I look at the patient's age, region of pain, area that they had the problem. Then immediately start thinking, 'well what is it likely to be', just based on the symptomology. so by the time I finish the history I think 'O.K, I think this persons got either, a rotator cuff tendonopathy, or it could be referred [pain]'. (P2)

Recognising relationships between clinical cues, such as specific clinical signs and symptoms, meant that diagnoses could be verified and confirmed through further clinical assessment, enabling participants to construct a diagnosis more efficiently, in less time and with less effort. Participants appeared to 'clump' chunks of clinical information together to arrive at a diagnosis, which could be verified or modified through further clinical examination. If a pattern was recognised, participants could immediately evaluate and test their hypotheses:

My mind might be thinking this is a typical facet joint problem.so I will go through the procedure of looking above and below and those joint closer to examine them. (P1)

Within the first few minutes, well a minute of him talking, I felt the lesion [diagnosis] was the most probable. And I was looking for other reasons thereafter, to see whether it could have been anything else. [but] quite frankly I just knew what it was and I can't think what else it could be. (P11)

For most participants the nature of a diagnosis centred on a mechanical, physical and objective entity that lay within the patient's body:

I think in every osteopathic patient, you end up with a diagnosis which is some kind of tissue causing symptom type of scenario. (P9)

.if the patients' problem is due to osteoarthritis of the hip, then you must appreciate that the osteoarthritis has caused a shortening of the hip musculature and therefore you have to maintain as much movement as possible in the hip region and remove their pain. (P1)

.manual provocation techniques to reproduce their [patients'] symptoms are some of the best diagnostic tools. If I can find the exact action that produces pain then I can have an idea of what kind of tissue is involved and start to think about treatment. (P5)
However, several participants considered and explored patients' pain and dysfunction in the context of their family, work and social life during their diagnostic reasoning:

I like to see the other factors that would be influencing the way that they [the patient] experience their problem.it gives you a much rounder picture of the person you're treating. (P6)

I pay quite a lot of attention to how a patient has pain throughout the day and throughout their life.[and] pain to me is what the patient says it is, I'm really less concerned with optimal functioning of biomechanics and optimal symmetry.l'm more concerned with what the patient's pain means to them. (P3)

These participants commented on the importance of considering the multiple factors which impact patients' pain and disability, and suggest an emphasis on the psychological and social aspects of the patient when constructing a diagnosis.

For all participants, diagnostic reasoning constituted a safety procedure whereby they ruled out a serious cause of their patients' problem. Participants showed flexibility in their diagnostic reasoning and could move between hypothetico-deductive and pattern recognition approaches. Hypothetico-deductive reasoning would be taken when confronted with an unfamiliar or complex problem, so that participants could deliberately and carefully collect clinical data and deductively generate multiple possible diagnoses to be further tested. Alternatively, when faced with a familiar or simple patient presentation, participants could draw on their previous experiences and recognise meaningful patterns and relationships between symptoms, enabling them to inductively build a diagnosis quickly and effi. The movement between hypothetico-deductive reasoning and pattern recognition appeared to be linked by the practitioners' awareness of, and refl upon their own diagnostic reasoning in the context of the presenting patient and the clinical situation. The comments below provide some examples of participants moving between hypothetico-deductive reasoning and pattern recognition:

If somebody is exhibiting signs that they're not improving I'll sit back and re-think about it, [and] really go through the process again.it's almost taking the process into a much more focused and logical process than by doing it by intuition. (P5) 


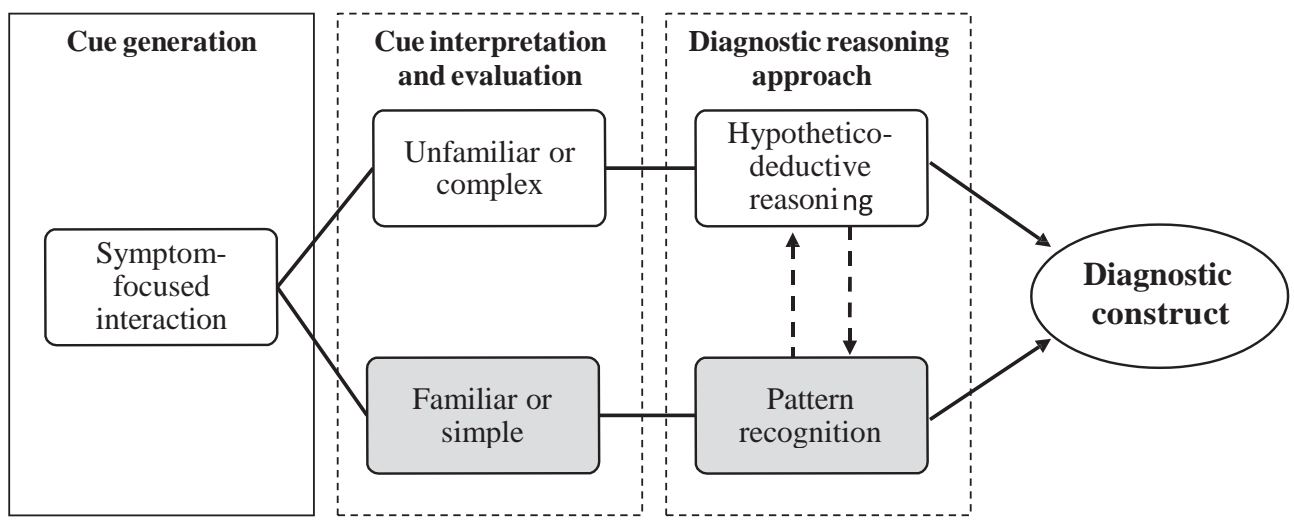

Figure 1 Interaction between hypothetico-deductive reasoning and pattern recognition.

If things [symptoms] hadn't improved, I would then have gone into a much more in-depth analysis of the intervertebral. (P10)

The minute anything happens, something unusual.you train yourself to flick up into the conscious level [when] things that are aberrant or unusual and require further investigation. (P6)

Fig. 1 above illustrates how participants would adopt either a hypothetico-deductive approach to diagnostic reasoning if the patient presented with symptoms which were complex of unfamiliar. If patients presented with simpler or familiar symptoms, participants could recognise these as "patterns' and inductively reason to construct a diagnosis.

\section{Discussion}

To our knowledge, this is the first published research investigating the diagnostic reasoning of osteopaths. The findings indicate that the diagnostic reasoning of experienced osteopaths involved the interaction of hypothetico-deductive reasoning and pattern recognition approaches. These findings support models of diagnostic reasoning proposed theoretically in the osteopathic literature. ${ }^{43,44,58}$ The study found that when formulating a diagnosis all participants adopted two cognitively based diagnostic reasoning approaches, consistent with the medical literature, namely hypothetico-deductive reasoning ${ }^{19}$ and pattern recognition. ${ }^{59}$ In this study, there was interplay of hypotheticodeductive reasoning and pattern recognition, as a result of the perceived level of complexity and degree of familiarity of the patient presentation. This is the first study to indicate an interaction of diagnostic reasoning approaches in relation to osteopathy, and supports existing research in physiotherapy $2,8,60,61$ and occupational therapy, ${ }^{10,30}$ that experienced practitioners are able to move flexibly between diagnostic reasoning approaches depending on the nature of the clinical situation.

The conditions under which both diagnostic reasoning approaches occur, is also consistent with the literature on diagnostic reasoning ${ }^{57,62}$ and more general theories of human cognition and decision-making, namely 'Dual Processing Theory'63e65 and 'Cognitive Continuum Theory'. ${ }^{66,67}$ Under conditions of familiarity, whereby the experienced practitioners recognised characteristics of the patient (for example, age and general appearance), and characteristics of their illness presentation (for example, the location and severity of pain, and associated symptoms), they recognised patterns and relationships between different cues. This enabled practitioners to formulate early diagnostic impressions and hypotheses based on the similarity between the present situation and previous experience, thereby inductively building diagnoses. This finding suggests that experienced osteopaths possessed a well organised knowledge base ${ }^{28}$ and countless similar exemplars, ${ }^{49}$ which they could 'activate' immediately and automatically. This inductive approach to diagnostic reasoning is considered to be a central element of clinical reasoning expertise. $8,61,68,69$

However, during unfamiliar conditions or when faced with a complex clinical presentation, participants in this study, deliberately and consciously used a hypothetico-deductive approach to diagnostic reasoning. ${ }^{19} \quad$ Hypothetico-deductive reasoning involved practitioners systematically acquiring and validating cues through a deliberate process of 'observation and measurement' (i.e. further clinical examination) so that hypotheses 
could be rejected, accepted of refined. The finding that osteopaths in this study re-examined patients and re-evaluated their diagnoses under conditions of uncertainty or complexity, such as when a patient failed to respond to treatment as predicted or if their symptoms worsened, is congruent with models of diagnostic reasoning developed from research ${ }^{2,8}$ and proposed theoretically ${ }^{70 e 72}$ in musculoskeletal physiotherapy.

The findings from this study support the existing theoretical and research literature that metacognitive processes form the link between hypothetico-deductive reasoning and pattern recognition. ${ }^{2,73,74}$ All participants in this study demonstrated the capability to reflect on their diagnostic reasoning processes and move between different reasoning approaches. Although this finding is consistent with existing literature which argues that metacognitive skills are associated with well-developed clinical reasoning capabilities, ${ }^{2,21,59}$ this is the first study to explore metacognition relation to osteopathic clinical reasoning. When constructing a diagnosis, participants reflected on the nature of the situation, such as its complexity and familiarity, and monitored their diagnostic reasoning processes. This finding concurs with Eraut who posits that when practitioners are confronted with a problem, they rapidly read the situation and proceed in a state of "continued alertness". ${ }^{75, p .15}$ This suggests that for the experienced osteopaths in this study, metacognitive and reflective processes are fundamental to enabling them to safely negotiate the complex situations encountered in professional practice and facilitate their development of a diagnosis and is congruent with the clinical reasoning capabilities set out by the regulator in the UK. ${ }^{38}$

Osteopathic educators should incorporate strategies which nurture the development of students' critical reflection capabilities and enhance their problem solving skills so that they can become adaptable, reflective and thinking practitioners, able to navigate through a professional practice setting which is complex, uncertain, unstable, unique and value-laden. ${ }^{76}$ The educational skills of clinical tutors could be further developed so that they can work with students to emphasise learning from practice and decision-making, in order to promote an engaged and active learning process during clinical education. ${ }^{77}$

The findings also have implications for continual professional development (CPD). Current CPD may focus too heavily on the acquisition of technical skills (for example, advanced hands-on skills, additional treatment modalities), which are conducted away from practice. Such CPD courses are often short, and may not foster and promote critically reflective practice and the development of diagnostic reasoning and reflexivity skills. CPD should enable practitioners to learn from practice and increase their capacity to synthesise, blend and critically evaluate different sources of knowledge, including research evidence. As an alternative to short courses and CPD training, peer observation of practice ${ }^{78}$ may help practitioners to learn from and in practice and develop their diagnostic reasoning and metacognitive skills.

This study explored the diagnostic reasoning of experienced osteopaths, many of which held or had held educational positions in osteopathic education institutions in the UK. This limited number of perspectives from a limited number of individuals means that the transferability ${ }^{79}$ of the findings to the wider osteopathic profession needs to be established through further research. This is amongst the first research of its kind in osteopathy and there are a number of key areas of osteopathic clinical reasoning which warrant further exploration. For example, research could be carried out to explore the theoretical 'reach' of the findings to osteopaths with different levels of experience, in other countries or practitioners that work in other clinical settings, such as the National Health Service.

\section{Conclusion}

The findings from this study indicate that experienced osteopaths adopt hypothetico-deductive and pattern recognition approaches to diagnostic reasoning. Participants in this study demonstrated well-developed metacognitive skills, which enabled them to move between two diagnostic reasoning approaches depending on the nature of the clinical problem and clinical situation. This study is amongst the first to provide researchbased knowledge of this specific and fundamental aspect of osteopathic clinical reasoning. The findings from this study support the theoretical literature on diagnostic reasoning in osteopathy, and also suggest that osteopaths' diagnostic reasoning processes are akin to practitioners of other healthcare professions including medicine and musculoskeletal physiotherapy.

\section{Acknowledgements}

Thanks to the British College of Osteopathic Medicine, the British Naturopathic and Osteopathic Association and the Osteopathic Educational Foundation for their support of this doctoral research. 


\section{References}

1. Higgs J, Jones M, Edwards I, Beeston S. Clinical reasoning and practice knowledge. In: Higgs J, Richardson B, Dahlgren MA, editors. Developing practice knowledge for health professionals. Edinburgh: Butterworth Heinemann; 2004. p. 181e99.

2. Edwards I, Jones M, Carr J, Braunack-Mayer A, Jensen GM. Clinical reasoning strategies in physical therapy. Phys Ther 2004;84:312e30.

3. Thomson OP, Petty NJ, Moore AP. Clinical reasoning in osteopathy e more than just principles? Int J Osteopath Med 2011;14:71e6.

4. Unsworth C. Review of methodologies for researching clinical reasoning. In: Schell BAB, Schell JW, editors. Clinical and professional reasoning in occupational therapy. Philadelphia, Pa.; London: Lippincott Williams \& Wilkins; 2008. p. 371e400.

5. Arocha JF, Patel VL. Methods in the study of clinical reasoning. In: Higgs J, Jones $M$, Loftus S, Christensen N, editors. Clinical reasoning in the health professions. 3rd ed. Oxford: Elsevier Butterworth-Heinemann; 2008. p. 193 e203.

6. Loftus S, Smith M. A history of clinical reasoning research. In: Higgs J, Jones M, Loftus S, Christensen N, editors. Clinical reasoning in the health professions. 3rd ed. Oxford: Elsevier Butterworth-Heinemann; 2008. p. $205 e 12$.

7. Cruz EB, Moore A, Cross V. Clinical reasoning and patientcentred care in musculoskeletal physiotherapy in Portugal e a qualitative study. Man Ther 2012;17:246e50.

8. Doody C, McAteer M. Clinical reasoning of expert and novice physiotherapists in an outpatient orthopaedic setting. Physiotherapy 2002;88:258e68.

9. Rogers JC, Holm MB. Occupational therapy diagnostic reasoning: a component of clinical reasoning. Am J Occup Ther 1991;45:1045e53.

10. Mattingly $\mathrm{C}$. What is clinical reasoning? Am J Occup Ther 1991;45:979e86.

11. Unsworth CA. Using a head-mounted video camera to explore current conceptualizations of clinical reasoning in occupational therapy. Am J Occup Ther 2005;59:31(10).

12. Scholes J, Endacott R, Biro M, Bulle B, Cooper S, Miles M, et al. Clinical decision-making: midwifery students' recognition of, and response to, post partum haemorrhage in the simulation environment. BMC Pregnancy Childbirth 2012;12:19.

13. Tanner CA, Padrick KP, Westfall UE, Putzier DJ. Diagnostic reasoning strategies of nurses and nursing students. Nurs Res 1987;36:358e65.

14. Benner PE. From novice to expert: excellence and power in clinical nursing practice. Menlo Park, Calif.: AddisonWesley Pub. Co., Nursing Division; 1984.

15. Neufeld VR, Norman GR, Feightner JW, Barrows HS. Clinical problem-solving by medical students: a cross-sectional and longitudinal analysis. Med Educ 1981;15:315e22.

16. Barrows HS, Norman GR, Neufeld VR, Feightner JW. The clinical reasoning of randomly selected physicians in general medical practice. Clin Invest Med 1982;5:49e55.

17. Groot ADd. Thought and choice in chess, vol. 463. The Hague, Paris: Mouton \& Co.; 1965xvi.

18. Chase W, Simon HA. Perception in chess. Cogn Psychol 1973;4:55e81.

19. Elstein AS, Shulman LS, Sprafka SA. Medical problem solving: an analysis of clinical reasoning. Cambridge, Mass.; London: Harvard University Press; 1978.

20. Mylopoulos M, Regehr G. Putting the expert together again. Med Educ 2011;45:920e6.
21. Jensen GM, Gwyer J, Shepard KF. Expert practice in physical therapy. Phys Ther 2000;80:28e43 [discussion 44e52].

22. Jensen GM, Shepard KF. Expertise in physical therapy practice. Saunders Elsevier; 2006.

23. Petty NJ, Scholes J, Ellis L. The impact of a musculoskeletal masters course: developing clinical expertise. Man Ther 2011;16:590e5.

24. Norman G. Research in clinical reasoning: past history and current trends. Med Educ 2005;39:418e27.

25. Groen GJ, Patel VL. Medical problem-solving: some questionable assumptions. Med Educ 1985;19:95e100.

26. Barrows HS, Feltovich PJ. The clinical reasoning process. Med Educ 1987;21:86e91.

27. Patel VL, Groen GJ. The general and specific nature of medical expertise: a critical look. In: Smith J, Ericsson KA, editors. Toward a general theory of expertise: prospects and limits, vol. x. Cambridge: Cambridge University Press; 1991. p. 344

28. Schmidt HG, Norman GR, Boshuizen HP. A cognitive perspective on medical expertise: theory and implication. Acad Med 1990;65:611e21.

29. Jones M, Jensen $G$, Edwards. Clinical reasoning in physiotherapy. In: Higgs J, Jones M, Loftus S, Christensen N, editors. Clinical reasoning in the health professions. 3rd ed. Oxford: Elsevier Butterworth-Heinemann; 2008. p. $245 e 56$.

30. Fleming $M H$. Clinical reasoning in medicine compared with clinical reasoning in occupational therapy. Am J Occup Ther 1991;45:988e96.

31. Payton OD. Clinical reasoning process in physical therapy. Phys Ther 1985;65:924e8.

32. Thornquist E. Diagnostics in physiotherapy e processes, patterns and perspectives. Part I. Adv Physiother 2001;3: $140 \mathrm{e} 50$.

33. GOsC. General Osteopathic Council e snapshot survey. London: General Osteopathic Council; 2001. Available from: http: //www.osteopathy.org.uk/uploads/survey2snapshot_ survery_results_2001.pdf.

34. Fawkes C, Leach J, Mathias S, Moore AP. Standardised data collection within osteopathic practice in the UK: development and first use of a tool to profile osteopathic care in 2009. National Council for Osteopathic Research: University of Brighton; 2010.

35. Fryer G, Johnson JC, Fossum C. The use of spinal and sacroiliac joint procedures within the British osteopathic profession. Part 2: treatment. Int J Osteopath Med 2010; 13:152e9.

36. Johnson SM, Kurtz ME. Osteopathic manipulative treatment techniques preferred by contemporary osteopathic physicians. J Am Osteopath Assoc 2003;103:219e24.

37. Orrock P. Profile of members of the Australian Osteopathic Association: part 1 e the practitioners. Int J Osteopath Med 2009;12:14e24.

38. General Osteopathic Council. Osteopathic practice stan dards. London: General Osteopathic Council; 2012.

39. OBA. Osteopathy code of conduct. Osteopathy Board of Australia; 2011. http://www.osteopathyboard.gov.au/ Codes-Guidelines.aspx.

40. OCNZ. Capabilities for osteopathic practice, vol. 2012 The Osteopathic Council of New Zealand; 2010. Available from: http://www.osteopathiccouncil.org.nz/ competency-standards.html.

41. WHO. Benchmarks for training in osteopathy. Geneva: World Health Organization; 2010.

42. Sammut EA, Searle-Barnes PJ. Osteopathic diagnosis. Cheltenham: Stanley Thornes; 1998.

43. Smith AE. Osteopathic diagnosis. Br Osteopathic J 1971;5: $2 e 8$. 
44. Sprafka SA. Clinical problem solving. In: Ward R, editor. Foundations for osteopathic medicine. 2nd ed. Philadelphia: Lippincott Williams \& Wilkins; 2003. p. 257e79.

45. Smith M, Higgs J, Ellis E. Characteristics and processes of physiotherapy clinical decision making: a study of acute care cardiorespiratory physiotherapy. Physiother Res Int 2008;13:209e22.

46. Smith $M$, Higgs J, Ellis E. Factors influencing clinical decision making. In: Higgs $J$, Jones $M$, Loftus $S$, Christensen $\mathrm{N}$, editors. Clinical reasoning in the health professions. 3rd ed. Oxford: Elsevier Butterworth-Heinemann; 2008. p. 89e100.

47. Boshuizen HC, Schmidt $\mathrm{H}$. The development of clinical reasoning expertise. In: Higgs $J$, Jones $M$, Loftus $S$, Christensen $\mathrm{N}$, editors. Clinical reasoning in the health professions. 3rd ed. Oxford: Elsevier Butterworth-Heinemann; 2008. p. 113e21.

48. Custers EJ, Boshuizen HP, Schmidt HG. The influence of medical expertise, case typicality, and illness script component on case processing and disease probability estimates. Mem Cognit 1996;24:384e99.

49. Norman G, Young M, Brooks L. Non-analytical models of clinical reasoning: the role of experience. Med Educ 2007; 41:1140e5.

50. Fryer G. Special issue: osteopathic principles. Int J Osteopath Med 2013;16:1e2.

51. Thomson OP, Petty NJ, Moore AP. Clinical decision-making and therapeutic approaches in osteopathy $e$ a qualitative grounded theory study. Man Ther 2013. http://dx.doi.org/ 10.1016/j.math.2013.07.008.

52. Thomson OP, Petty NJ, Moore AP. A grounded theory study of the conceptions of practice in osteopathy: a continuum from technical rationality to professional artistry. Man Ther 2013. http://dx.doi.org/10.1016/j.math.2013.06.005.

53. Thomson OP, Petty NJ, Scholes J. Grounding osteopathic research e introducing grounded theory. Int $J$ Osteopath Med 2013. http: //dx.doi.org/10.1016/j.ijosm.2013.07.010.

54. Thomson OP. Clinical decision making and therapeutic approaches of experienced osteopaths. University of Brighton; 2013. Unpublished PhD thesis.

55. Charmaz K. Constructing grounded theory. London; Thousand Oaks, Calif.: Sage Publications; 2006.

56. Haw K, Hadfield M. Video in social science research: functions and forms. Routledge; 2011.

57. Patel VL, Groen GJ. Knowledge based solution strategies in medical reasoning. Cogn Sci 1986;10:91e116.

58. Smith AE. Osteopathic diagnosis: history taking. $\mathrm{Br}$ Osteopath J 1971;5:10e5.

59. Arocha JF, Patel VL, Patel YC. Hypothesis generation and the coordination of theory and evidence in novice diagnostic reasoning. Med Decis Making 1993;13:198e211.

60. Embrey DG, Guthrie MR, White OR, Dietz J. Clinical decision making by experienced and inexperienced pediatric physical therapists for children with diplegic cerebral palsy. Phys Ther 1996;76:20e33.
61. Smith $M$, Joy $H$, Ellis $E$. Effect of experience on clinical decision making by cardiorespiratory physiotherapists in acute care settings. Physiother Theory Pract 2010;26: 89 e99.

62. Elstein AS. Thinking about diagnostic thinking: a 30-year perspective. Adv Health Sci Educ Theory Pract 2009;14: $7 e 18$.

63. Croskerry P. Cognitive forcing strategies in clinical decisionmaking. Ann Emerg Med 2003;41:110e20.

64. Croskerry P. A universal model of diagnostic reasoning. Acad Med 2009;84:1022e8.

65. Evans JS. Dual-processing accounts of reasoning, judgment, and social cognition. Annu Rev Psychol 2008;59: $255 e 78$.

66. Hammond KR, Brehmer B. Quasi-rational and distrust: implications for international conflict. Human judgement and social interactions. New York: Rineholt and Winston; 1973.

67. Custers EJFM. Medical education and cognitive continuum theory: an alternative perspective on medical problem solving and clinical reasoning. Acad Med 2013. Publish Ahead of Print:10.1097/ACM.0b013e31829a3b10.

68. Benner PA, Tanner CA, Chesla CA. Expertise in nursing practice: caring, clinical judgement and ethics. New York: Springer Pub. Co; 1996.

69. Jensen G, Rensik L, Haddad A. Expertise and clinical reasoning. In: Higgs J, Jones $M$, Loftus $S$, Christensen $\mathrm{N}$, editors. Clinical reasoning in the health professions. 3rd ed. Oxford: Elsevier Butterworth-Heinemann; 2008. p. $123 e 35$.

70. Petty NJ. Neuromusculoskeletal examination and assessment: $a$ handbook for therapists. Elsevier Health Sciences UK; 2011.

71. Jones MA. Clinical reasoning in manual therapy. Phys Ther 1992;72:875e84.

72. Maitland GD, Hengeveld E, Banks K, English K. Maitland's vertebral manipulation. Elsevier Butterworth-Heinemann; 2005.

73. Mamede S, Schmidt HG, Penaforte JC. Effects of reflective practice on the accuracy of medical diagnoses. Med Educ 2008;42:468e75.

74. Croskerry P. Clinical cognition and diagnostic error: applications of a dual process model of reasoning. Adv Health Sci Educ Theory Pract 2009;14:27e35.

75. Eraut M. Schon shock: a case for refraining reflection-inaction? Teach Teach Theory Pract 1995;1:9e22.

76. Schön DA. The reflective practitioner: how professionals think in action. Nueva York, EUA: Basic Books; 1983.

77. Spencer JA, Jordan RK. Learner centred approaches in medical education. Br Med J 1999;318:1280.

78. Petty NJ, Morley M. Clinical expertise: learning together through observed practice. Man Ther 2009;14:461e2.

79. Bryman A. The nature of qualitative research. In: Bryman A, editor. Social research methods. 3rd ed. Oxford: Oxford University Press; 2008. p. 365e99. 